\title{
Search for isospin effects on nuclear level density
}

\author{
A. Brondi ${ }^{1}$, A. Di Nitto ${ }^{1}$, G. La Rana ${ }^{1, a}$, R. Moro ${ }^{1}$, P. N. Nadotchy ${ }^{2}$, E. Vardaci ${ }^{1}$, A. Ordine ${ }^{1}$, A. Boiano ${ }^{1}$, M. Cinausero $^{3}$, \\ G. Prete ${ }^{3}$, V. Rizzi ${ }^{3}$, N. Gelli ${ }^{4}$, F. Lucarelli ${ }^{4}$ \\ 1 Istituto Nazionale di Fisica Nucleare and Dipartimento di Scienze Fisiche, Universitá di Napoli "Federico II",Via Cinthia, \\ 80126 Napoli, Italy \\ 2 Istituto Nazionale di Fisica Nucleare, Via Cinthia, 80126 Napoli, Italy \\ 3 Laboratori Nazionali di Legnaro dell'Istituto Nazionale di Fisica Nucleare, Legnaro (Padova), Italy \\ 4 Istituto Nazionale di Fisica Nucleare and Dipartimento di Fisica, Firenze, Italy
}

\begin{abstract}
Studies on the isospin dependence of the level density have been recently reported in the literature for nuclei with $20 \leq \mathrm{A} \leq 110$. Corrections to the level density have been deduced which would imply a significant reduction of this quantity for nuclei far from the valley of stability. Isospin effects on the level density are also expected through the symmetry energy contribution to the nuclear masses, which is predicted to increase with the temperature. According to these findings, we have implemented the statistical model in order to account for isospin effects on the level density parameter $a$ and on the temperature-dependent symmetry energy. We present the results of calculations for the decay of a variety of neutron-rich composite systems. We found that isospin produces sizable effects on different observables, this result being promising for future experiments with the second generation RIB facilities SPES and SPIRAL2. We report the results of a first experiment aimed at searching for isospin effects in the decay of ${ }^{139} \mathrm{Eu}$ composite nuclei produced by a stable beam at $\mathrm{E}_{x}=90 \mathrm{MeV}$.
\end{abstract}

\section{Introduction}

Nuclear level density is a fundamental quantity in many aspects of nuclear reactions and in particular in nuclear astrophysics as well as in the search for superheavy elements. Much work has been devoted to study this quantity whose most commonly used expression is based on the Fermi-gas model. At present, the level density has only been studied in nuclei close to the valley of stability and mainly on the neutron-deficient side. Starting from the Bethe work [1] , based on an energy independent density of single particle states, angular momentum as well as pairing and shell effects have been included in more realistic expressions of the level density. In all these studies, isospin effects have been usually neglected, as they are expected to be relatively small for nuclei close to the valley of stability, as those produced by stable beams. Isospin is expected to affect two quantities: the level density parameter $a$ and the symmetry energy. Experimentally, these effects would appear as a change in the particle multiplicity and energy spectra as well as in the relative yields of the compound nucleus decay channels. In particular, level densities can be deduced from these observables on the base of the statistical model.

\section{Isospin effects on the level density parameter $a$}

The level density parameter $a$ has been the subject of many investigations at low and high excitation energy. The evolution of this parameter from the low temperature values, typically $\mathrm{A} / 8.5 \mathrm{MeV}^{-1}$, toward that predicted by the Fermigas model, $\mathrm{A} / 15 \mathrm{MeV}^{-1}$, is supported by several observations [2]. With this description of the level density, the statistical model proved to be able to reproduce most of the data of studied reactions with stable beams, although inadequacies of the Fermi gas form have been indicated by different authors [3], [4], [5]. In the framework of the Fermi-gas model the isospin effects on the level density can be taken into account including a factor related to the isospin component $\mathrm{T}_{3}=(\mathrm{N}-\mathrm{Z}) / 2[6]$ as well as to the density of single-particle states at the Fermi energy and the excitation energy of the system. According to this, the level density is expected to decrease with increasing $\left|\mathrm{T}_{3}\right|$. Recently, the analysis of the level density data [7] for nuclei with 20 $\leq \mathrm{A} \leq 110$ [8], [9] has been carried out comparing different isospin dependences. It has been shown that, compared to the prescription based on the $\mathrm{N}-\mathrm{Z}$ dependence, a reduction factor of the level density based on the distance from the valley of stability Z-Zo, where Zo is the atomic number of the beta stable isotope with the same mass, provides a better description of data. This result brings evidence that the level density parameter has dependence on $\mathrm{N}$ and $\mathrm{Z}$ rather than just on A. These predictions, which would have strong implications for nuclei far from the stability line, are sup-

\footnotetext{
a e-mail: giovanni.larana@na.infn.it
} 
ported by recent experimental findings [10], [11] and realistic calculations [12] of level density of nuclei far from the stability line. Based on the realistic assumptions that the levels of the compound system for nuclei far from the stability line have widths which are large enough to remove them from the category of compound nucleus levels, a strong reduction of the level density is obtained.

\subsection{Search for clues to isospin effects in the decay of ${ }^{139} \mathrm{Eu}$ composite nuclei at $\mathrm{E}_{x}=90 \mathrm{MeV}$}

Although isospin effects are expected to be relevant for exotic nuclei, the statistical model predicts that they could be observable also for particular nuclei close to the valley of stability. With aim to search for clues to isospin effects, we have measured the evaporative charged particles from the decay of the composite system ${ }^{139} \mathrm{Eu}$, produced by $180 \mathrm{MeV}{ }^{32} \mathrm{~S}+{ }^{107} \mathrm{Ag}$ reaction at $\mathrm{E}_{x}=90 \mathrm{MeV}$. The experiment was performed at the Tandem-ALPI complex of the Laboratori Nazionali di Legnaro (LNL). We used the $8 \pi \mathrm{LP}$ apparatus [13] to detect light charged particles (LCPs) in coincidence with evaporation residues (ERs). These latter have been detected by means of four Parallel Plate Avalanche Counter modules. In a separate experiment ER cross section has been measured by means of the electrostatic deflector of LNL [14]. We have compared proton and alpha particle energy spectra as well as the ERLCP angular correlations to the predictions of the statistical model, where N-Z and Z-Zo isospin dependence of the level density has been included according to Ref. [8], [9]. ER-proton angular correlation are shown in Fig. 1 for protons detected in the BALL section of $8 \pi \mathrm{LP}$, in coincidence with ER at $\theta_{l a b}=4.5^{\circ}$ in the vertical plane, below the beam. The BALL detector number in the figure increases going from backward to forward angles along the rings of $8 \pi \mathrm{LP}$, each of them being positioned at a fixed angle theta and with detectors spanning azimuthal angles phi from 0 to $2 \pi$.

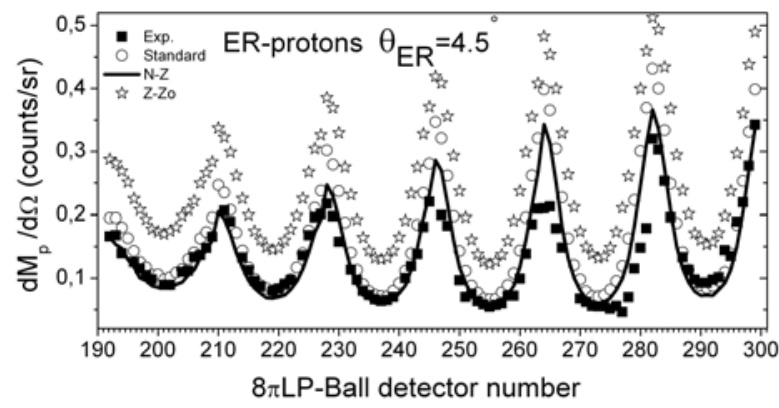

Fig. 1. Experimental angular correlation ER-proton for $180 \mathrm{MeV}$ ${ }^{32} \mathrm{~S}+{ }^{107} \mathrm{Ag}$ system, compared with the predictions of the statistical model.

The observed oscillating behavior is due to a combined effect of kinematics and angular momentum. Data are com-

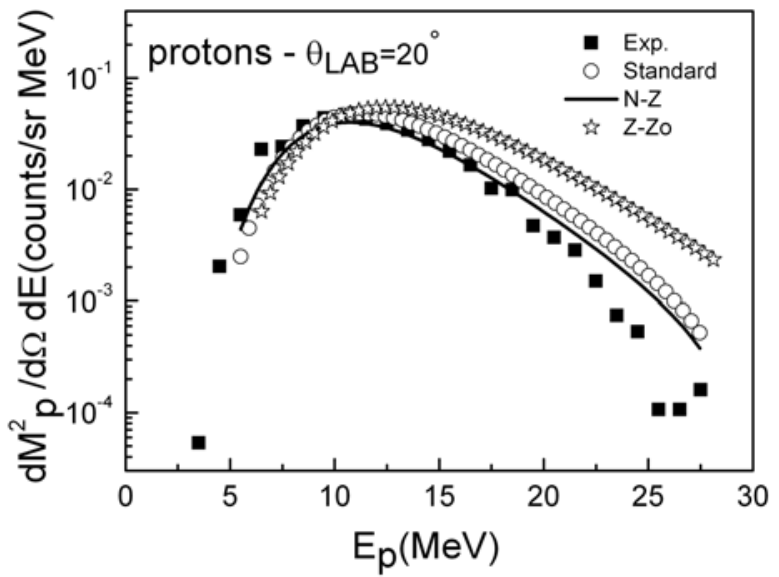

Fig. 2. Experimental proton spectrum for $180 \mathrm{MeV}{ }^{32} \mathrm{~S}+{ }^{107} \mathrm{Ag}$ system, compared with the predictions of the statistical model.

pared with statistical model calculations assuming three prescriptions: no isospin, $\mathrm{N}-\mathrm{Z}$ and Z-Zo dependence of level density. As is can be seen, the Z-Zo dependence is not able to reproduce the data. An overall reasonable agreement is obtained with the standard and the N-Z option, although the latter is slightly more supported by the data. The same behavior is observed for the energy spectra, as it is shown in Fig. 2 where the proton spectrum at $\theta_{L A B}=20^{\circ}$ is shown. This result, which is not confirming the findings of Al Quaishi et al. [8], [9] could be related to: i)the relatively high excitation energy involved in this system, which is expected to wash out the isospin effects and to ii)the values of the parameters used in our calculation to modulate the Z-Zo dependence, which have been kept equal to those extracted in Ref. [8] at low excitation energy. A comprehensive study of the isospin dependence of the level density will be possible for neutron-rich nuclei, using radioactive beams as those that will be provided by SPES and SPIRAL2 facilities. In order to evaluate to what extent such effects could be observed, statistical model calculations for different systems have been carried out. The results are reported in the next section.

\subsection{Statistical model predictions for neutron-rich nuclei}

As previously mentioned, low excitation energies are more suited for the study of isospin effects, as they limit the contribution of particle emission from nuclei closer to the valley of stability. Furthermore, decay channels involving a small number of particles provide a stringent test of the statistical model, the decay not being integrated over many particle steps. In particular, the analysis involving only one neutron emission allows a direct measurement of the level density. As an example of possible experiments, the channels $1 \mathrm{n}$ and $2 \mathrm{n}$ could be studied bombarding a ${ }^{4} \mathrm{He}$ target with medium-mass neutron-rich projectiles. These reactions meet the conditions of providing a high fusion cross 
section with a low excitation energy $(\simeq 20 \mathrm{MeV})$ of the composite systems. Furthermore, the relatively low angular momenta and excitation energies involved in these reactions are expected to enhance the effects of the isospin on the level density. In order to evaluate these effects we have carried out statistical model calculations using the code Lilita_N97 [15] for some neutron-rich composite nuclei at $\mathrm{E}_{x}=20 \mathrm{MeV}$, whose production will be possible by SPES and SPIRAL2 using a ${ }^{4} \mathrm{He}$ target. We show in Fig.3

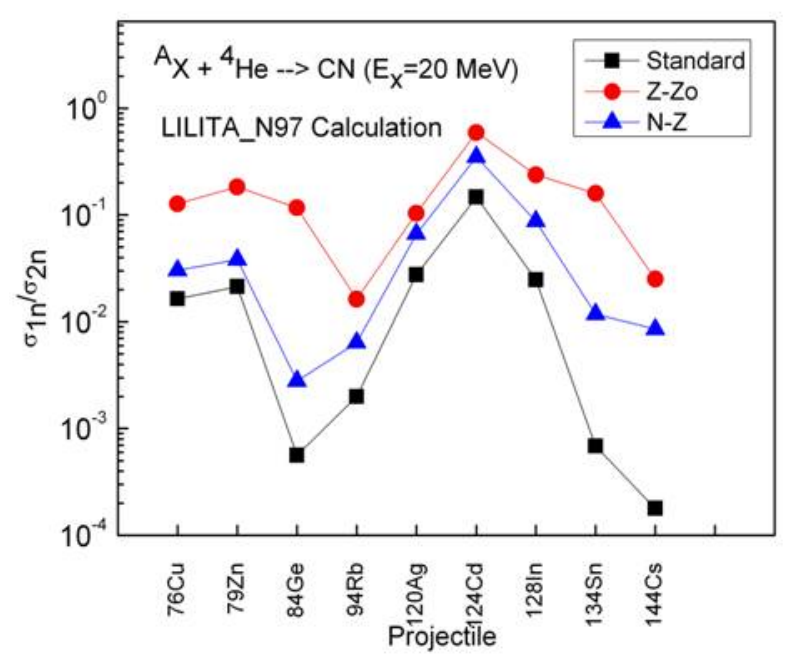

Fig. 3. Ratio between $1 \mathrm{n}$ and $2 \mathrm{n}$ channel cross sections predicted by the statistical model for different isospin dependences of the level density

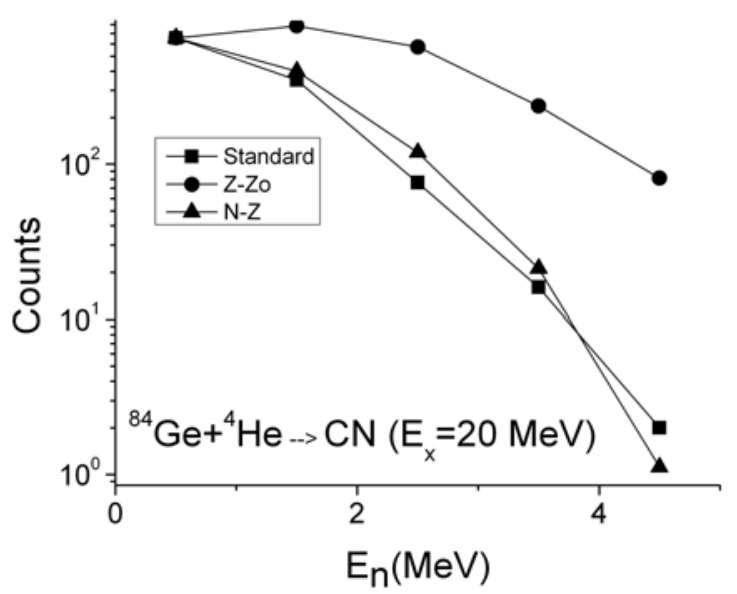

Fig. 4. Neutron spectra predicted by the statistical model for different isospin dependences of the level density. Spectra are normalized to the maximum.

the calculated ratio between $1 \mathrm{n}$ and $2 \mathrm{n}$ channel cross sections as a function of the projectile, taking into account the
$\mathrm{N}-\mathrm{Z}$ and Z-Zo dependence in the level density. The neutron energy spectrum for the reaction ${ }^{84} \mathrm{Ge}+{ }^{4} \mathrm{He}$ is shown in Fig.4. The calculation with no isospin (standard) is also reported for comparison. Significant effects are observed, indicating that the evaporative neutrons are a powerful tool for such a study. With the increase of the excitation energy, channels with a larger number of neutrons will open, and a reduction of isospin effects will result. The effects are still observables at $\mathrm{E}_{x}=80 \mathrm{MeV}$ in the energy spectra and in the yields of the evaporation residues.

\section{Isospin effects on the symmetry energy}

The effective nucleon mass $\mathrm{m} *$ is expected to decrease with increasing temperature for $\mathrm{T} \leq 2 \mathrm{MeV}$. This implies, apart from the decrease of the level density parameter $a \propto \mathrm{m}^{*}$, an increase with the temperature of the symmetry (kinetic) energy contribution to the nuclear binding energy through the dependence on the isospin component [16],[17],[18]: $\mathrm{E}_{\text {sym }}(\mathrm{T})=\mathrm{b}_{\text {sym }}(\mathrm{T}) .(\mathrm{N}-\mathrm{Z})^{2} / \mathrm{A}$. Variations of the symmetry energy have strong implications in nuclear astrophysics as they change the rate of electron capture in a collapsing star, and this in turn changes the energy of the final supernova explosion. It has been shown that the gain in the explosion may be comparable to the explosion energy itself [16].
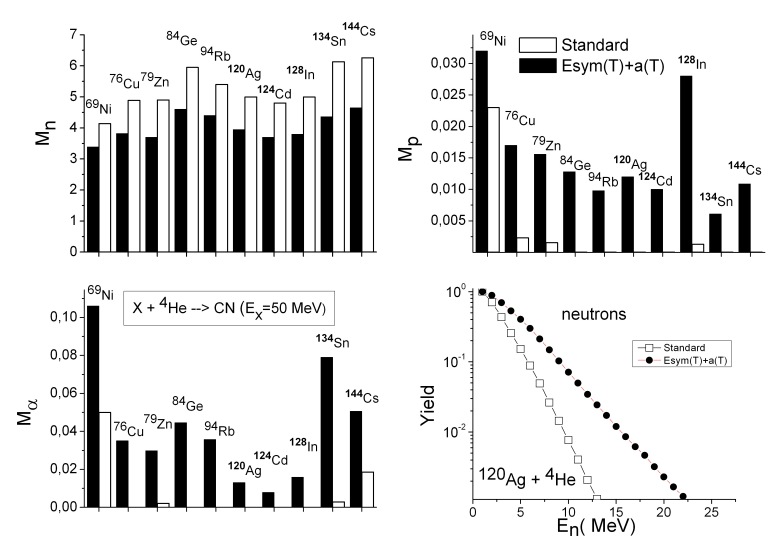

Fig. 5. Light particle multiplicities and neutron energy spectra predicted by the statistical model including temperaturedependent symmetry energy $\mathrm{E}_{\text {sym }}(\mathrm{T})$ and level density parameter $a(\mathrm{~T})$ according to Ref. [16],[17], respectively. The results of the standard calculation are also shown for comparison.

Experimentally, the effects related to the symmetry energy would appear as a change in the particle multiplicity as well as in the relative yields of the compound nucleus decay channels, as they affects the binding energies of the evaporative light particles. As this effect is of the order of a few $\mathrm{MeV}$ in each step of the evaporative chain, relatively low excitation energies are required in order to observe the effect and perform a test of the current models. We have included in the statistical model the symme- 
try energy prescription of Ref. [16] as well as the temperature dependence of the level density parameter $a$, obtained in the same theoretical framework [17]. Calculations for the same systems presented in the previous section, but at $\mathrm{E}_{x}=50 \mathrm{MeV}$, have been carried out. Predicted multiplicities for neutron, proton and alpha particles are shown in Fig. 5 for the standard option and including the symmetry energy as well as the temperature dependence of $a$ parameter (indicated by $\mathrm{E}_{\text {sym }}(\mathrm{T})+a(\mathrm{~T})$ on the figure). We obtain a strong sensitivity of the results on the two options. In particular, $\mathrm{E}_{\text {sym }}(\mathrm{T})+a(\mathrm{~T})$ option promotes the opening of channels involving LCPs, which otherwise would be closed assuming the standard prescription. A significant sensitivity is also observed for the neutron energy spectrum, shown in the figure, as well as for proton and alpha particle spectra and the yields of the ERs. It must considered that the effects of the symmetry energy on the evaporative process are rather general and different from those produced by the isospin effects on the level density parameter $a$, allowing to unfold the two effects.

\section{Conclusions and perspectives}

Level density of exotic nuclei are expected to be significantly affected by the isospin degree of freedom, through the level density parameter $a$ and the symmetry energy. Statistical model calculations show that fusion-evaporation reactions are a good probe for studying the level density of neutron-rich nuclei, as those that will be produced by SPES and SPIRAL2 facilities. In particular, ER-LCP angular correlations, LCP energy spectra and multiplicities as well as ER yields are expected to be good observables. The selection of decay channels involving a small number of particles appears to be crucial to enhance the observability of the isospin effects. This selection requires the identification of the ER or of its characterizing discrete gamma rays, which could be performed by VAMOS and/or EXOGAM (or AGATA) spectrometers at GANIL, respectively. The neutron detector NEDA, whose prototype is under construction within the preparatory phase of SPIRAL2, as well as the LCP detector DIAMANT should be used for light particle detection. Finally, ER-LCP angular correlations as well as fusion cross sections could be measured with $8 \pi \mathrm{LP}$ apparatus and the electrostatic separator at LNL, respectively. For the near future, developments of the statistical model in order to combine the effects on the level density parameter $a$ and on the symmetry energy are in progress, together with the inclusion of more realistic level densities in the model.

\section{References}

1. H.A. Bethe, Phys. Rev. 50, (1936) 332.

2. See for example G. Nebbia et al., Phys. Lett. B176, (1986) 20

3. S. M. Grimes, Phys. Rev. C42, (1990) 2744.

4. M. G. Mustafa et al., Phys. Rev. C45, (1992) 1078.

5. A. V. Voinov et al., Phys. Rev. C76, (2007) 044602.
6. H. Feshbach "Theoretical Nuclear Physics-Nuclear Reactions" J. Wiley 1992.

7. M.R. Bhat, in Evaluated Nuclear Structure Data File (ENSDF), Nuclear Data for Science and Technology, edited by S.M. Qaim (Springer-Verlag, Berlin, 1992) p. 817.

8. S. I. Al-Quaraishi et al., Phys. Rev. C63, (2001) 065803.

9. S. I. Al-Quaraishi et al., Phys. Rev. C 67, (2003) 015803.

10. U. K. Pal et al., J. Phys. G 25, (1999) 1671.

11. B. V. Zhuravlev et al., Phys. of Atomic Nuclei 69, (2006) 363.

12. S. M. Grimes et al., AIP Conf. Proc. 1005, (2008) 57.

13. E. Fioretto et al., IEEE Trans. Nucl. Scie. 44, (1997) 1017.

14. S. Beghini et al., Nucl. Instrum. Methods A 239, (1985) 585.

15. LilitaN_97 is an extensively modified version of the original Lilita program made by J. Gomez del Campo and R. G. Stockstad, Oak Ridge National Laboratory, (Rep. No TM7295, 1981 unpublished).

16. P. Donati et al., Phys. Rev. Lett. 72, (1994) 2835.

17. W.E. Ormand et al., Phys. Rev. C 40, (1989) 1510.

18. D. J. Dean et al., Phys. Rev. C 66, (2002) 045802. 